\section{Commentary: Pulmonary vein stenosis: Not just a surgical solution}

\author{
Harold M. Burkhart, MD, ${ }^{\mathrm{a}}$ and \\ Heather N. Anderson, MD ${ }^{\mathrm{b}}$
}

Pulmonary vein stenosis (PVS) is poorly understood and often progressive with little treatment consensus. Perhaps the most important contribution to the management of PVS was the introduction of the sutureless repair for postrepair PVS more than 2 decades ago. ${ }^{1,2}$ The ability to create a large anastomosis, avoid pulmonary vein manipulation, and reduce the risk for distortion are a few of the advantages that make this technique appealing. Several reports in the literature have demonstrated success with this technique. ${ }^{3,4}$ Most recently, Kalfa and colleagues ${ }^{5}$ reported a multi-institutional experience demonstrating that the sutureless repair was an independent protective factor against pulmonary vein restenosis. Despite the surgical advances, there are patients who continue down a progressive PVS disease pathway resulting in death or need for lung transplant. This substantiates the idea that PVS needs more than a just a surgical solution.

Vanderlaan and colleagues ${ }^{6}$ present a comprehensive expert opinion primarily focused on the management of PVS in pediatric patients. The authors highlight the typical progressive and relentless nature of PVS in children compared with the more indolent course described in adults. This is particularly true of children with described risk factors for progressive PVS, including early age at onset, multivessel disease, prior pulmonary vein intervention, residual

\footnotetext{
From the ${ }^{\mathrm{a}}$ Division of Cardiovascular and Thoracic Surgery, and ${ }^{\mathrm{b}}$ Section of Pediatric Cardiology, University of Oklahoma Health Sciences Center, Oklahoma City, Okla.

Disclosures: The authors reported no conflicts of interest.

The Journal policy requires editors and reviewers to disclose conflicts of interest and to decline handling or reviewing manuscripts for which they may have a conflict of interest. The editors and reviewers of this article have no conflicts of interest.

Received for publication June 23, 2020; accepted for publication June 24, 2020; available ahead of print June 30, 2020.

Address for reprints: Harold M. Burkhart, MD, Division of Cardiovascular and Thoracic Surgery, University of Oklahoma Health Sciences Center, PO Box 26901, WP-2230, Oklahoma City, OK 73105 (E-mail: Harold-burkhart@ouhsc. edu).

J Thorac Cardiovasc Surg 2021;161:2177-8

$0022-5223 / \$ 36.00$

Copyright (c) 2020 by The American Association for Thoracic Surgery

https://doi.org/10.1016/j.jtcvs.2020.06.066
}

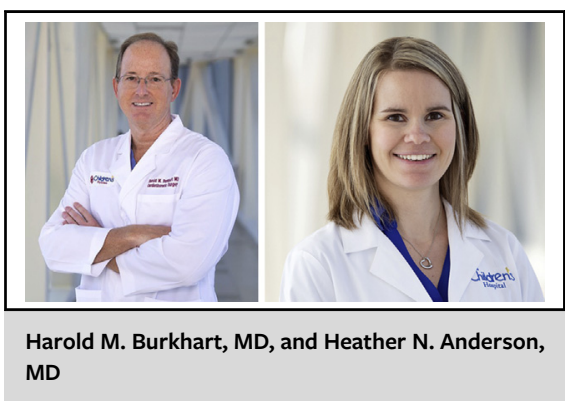

CENTRAL MESSAGE

Although advances in surgical techniques have been helpful in the treatment of pulmonary vein stenosis, a multidisciplinary, multimodality approach is essential.

PVS after repair, and history of prematurity. This article points out that it is essential to understand which patients are at risk of PVS to be diligent about surveillance in these patients. In addition, gaining insight into the underlying biological mechanisms that lead to progressive PVS is critical in helping guide future therapies.

Key takeaway points include:

- Early diagnosis is essential. Studies of PVS suggest that there may be upstream propagation of intimal proliferation and stenosis when downstream obstruction is present, underscoring the importance of aggressive monitoring protocols and prompt intervention to prevent disease progression.

- No magic bullet treatment is available. All interventions - catheter-based, surgical, and experimental medical therapies-have shown limited benefit in patients at high risk for progression of PVS.

- It takes a team. An institutional multidisciplinary team is needed to monitor, treat, and follow these at-risk patients. In addition, development of treatment protocols as well as multi-institutional collaboration are undoubtedly part of the solution.

The authors present an excellent, current overview of PVS. Although much progress has been made in recognizing the problem and improving therapies, much work still needs to be done. What this opinion piece is lacking will be evident to readers: A consensus treatment protocol for these at-risk children. We have no doubt that through the work of these experts and the PVS Network (www.PVSNetwork.org), consensus practice guidelines are forthcoming. 


\section{References}

1. Lacour-Gayet F, Rey C, Planche C. Pulmonary vein stenosis. Description of a sutureless surgical procedure using the pericardium in situ [article in French]. Arch Mal Coeur Vaiss. 1996;89:633-6.

2. Najm HK, Caldarone CA, Smallhorn J, Coles JG. A sutureless technique for the relief of pulmonary vein stenosis with the use of in situ pericardium. J Thorac Cardiovasc Surg. 1998;115:468-70.

3. Yun TJ, Coles JG, Konstantinov IE, Al-Radi OO, Wald RM, Guerra V, et al. Conventional and sutureless techniques for management of the pulmonary veins: evolution of indications from postrepair pulmonary vein stenosis to primary pulmonary vein anomalies. J Thorac Cardiovasc Surg. 2005;129: 167-74.

4. Shi G, Zhu Z, Chen J, Ou Y, Hong H, Nie Z, et al. Total anomalous pulmonary venous connection: the current management strategies in a pediatric cohort of 768 patients. Circulation. 2017;135:48-58.

5. Kalfa DK, Belli E, Bacha E, Lambert V, di Carlo D, Kostolny M, et al. Outcomes and prognostic factors for postsurgical pulmonary vein stenosis in the current era. J Thorac Cardiovasc Surg. 2018;156:276-86.

6. Vanderlaan RD, Rome J, Hirsch R, Ivy D, Caldarone CA. Pulmonary vein stenosis: treatment and challenges. J Thorac Cardiovasc Surg. 2021;161:2169-76.
See Article on page 2169.

\section{Commentary: This commentary was challenging and relentless}

\author{
Charles B. Huddleston, MD
}

Most reports on the subject pulmonary vein stenosis (PVS) begin with the admonition that this disease is challenging or relentless and Vanderlaan and colleagues ${ }^{1}$ remind us once again of this simple fact. According to the Society of Thoracic Surgeons Congenital Heart Surgery database, there are approximately 150 operations performed annually in the United States and Canada for PVS in 115 centers. That means, of course, that most centers are performing 1 or 2 such operations annually. This underscores the importance of developing a network of interested parties to study this diagnosis. Vanderlaan and colleagues ${ }^{1}$ represent such a group and provide a valuable addition to the literature and summarize the knowledge to date. What exactly can we learn by reading their article?

- Do we know what causes PVS? The answer is no. Apart from some interesting theoretical genetic, embryologic, and mechanical propositions the etiology of PVS remains elusive.

From the St Louis University School of Medicine, St Louis, Mo.

Disclosures: The author reported no conflicts of interest.

The Journal policy requires editors and reviewers to disclose conflicts of interest and to decline handling or reviewing manuscripts for which they may have a conflict of interest. The editors and reviewers of this article have no conflicts of interest.

Received for publication June 13, 2020; accepted for publication June 14, 2020; available ahead of print June 29, 2020.

Address for reprints: Charles B. Huddleston, MD, St Louis School of Medicine, 1465 S Grand Blvd, St Louis, MO 63104 (E-mail: Charles.huddleston@health.slu.edu). J Thorac Cardiovasc Surg 2021;161:2178-9

$0022-5223 / \$ 36.00$

Copyright (c) 2020 by The American Association for Thoracic Surgery

https://doi.org/10.1016/j.jtcvs.2020.06.067

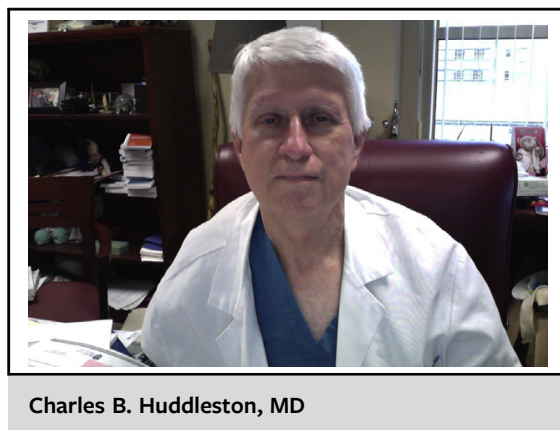

CENTRAL MESSAGE

Pulmonary vein stenosis is a challenging and relentless disease.

- Do we know how to make the diagnosis? The answer is yes! The recommendation is to start with an echocardiogram (no surprise there) and then move on to cardiac catheterization, computed tomography or magnetic resonance angiography and possibly nuclear medicine perfusion scans. That essentially runs the gamut of diagnostic tests available to heart specialists.

- Do we know how best to treat PVS? Sort of. Balloon dilatation and stenting of pulmonary veins were once considered hopeless and harmful. ${ }^{2}$ However, stents have made a comeback and drug-eluting stents appear better than bare-metal stents, recognizing that the largest drug-eluting stent is $5 \mathrm{~mm}$. What is the best operation? The so-called sutureless technique (it actually does require sutures) has better midterm results than other techniques such as patch venoplasty, ostial resection, reimplantation, or unroofing. However, the recurrence rate and mortality remain somewhat high. 